\title{
Relationship between inferior mesenteric artery diameter and rectal cancer
}

\author{
Feng $\mathrm{Chi}^{1}$, Shenkang Zhou ${ }^{1}$, Tienan $\mathrm{Bi}^{1}$, Wenjun Zhao ${ }^{2}$, Xiang Wang ${ }^{2}$
}

\author{
1Department of Gastrointestinal Surgery, Taizhou Hospital of Zhejiang Province, Zhejiang \\ University, Linhai, China \\ 2Department of Vascular Surgery, Taizhou Hospital of Zhejiang Province, Zhejiang University, \\ Linhai, China
}

Submitted: 26 July 2019; Accepted: 23 September 2019

Online publication: 25 March 2021

Arch Med Sci

DOI: https://doi.org/10.5114/aoms/112531

Copyright $\odot 2021$ Termedia \& Banach

\begin{abstract}
Introduction: A dilated inferior mesenteric vein has been reported in rectal cancer patients. However, no study has yet reported inferior mesenteric artery (IMA) enlargement in rectal cancer. We aimed to assess the relationship between the IMA diameter and rectal cancer.

Material and methods: Patients diagnosed with rectal cancer and a control group of 42 patients in our hospital from July 2017 to June 2019 were evaluated. The IMA diameter was independently measured by two observers on axial computed tomography images.

Results: The mean IMA diameter was wider in rectal cancer patients (2.49 $\pm 0.53 \mathrm{~mm})$ than in the control group $(2.20 \pm 0.47 \mathrm{~mm}, p<0.001)$. The IMA diameter of patients with stage I, stage II, stage III, and stage IV cancers was $2.24 \pm 0.36 \mathrm{~mm}, 2.45 \pm 0.39 \mathrm{~mm}, 2.80 \pm 0.55 \mathrm{~mm}$, and $2.85 \pm 0.51 \mathrm{~mm}$, respectively $(p<0.001)$. The IMA diameter correlated positively and moderately with TNM stage $(r=0.519, p<0.001)$. The IMA diameter of patients with T1, T2, T3, and T4 tumors was $2.18 \pm 0.31 \mathrm{~mm}, 2.39 \pm 0.50 \mathrm{~mm}, 2.55 \pm 0.48 \mathrm{~mm}$, and $2.73 \pm 0.51 \mathrm{~mm}$, respectively $(p<0.001)$. The IMA diameter also correlated positively and moderately with T stage $(r=0.457, p<0.001)$. The IMA diameter of patients with NO, N1, and N2 tumors was $2.37 \pm 0.39 \mathrm{~mm}, 2.83 \pm 0.60 \mathrm{~mm}$, and $2.71 \pm 0.40 \mathrm{~mm}$, respectively $(p<0.001)$; however, the IMA diameter did not correlate with $\mathrm{N}$ stage $(r=0.166, p=0.077)$. Patients with $\mathrm{M} 1$ tumors had a wider IMA diameter than patients with MO tumors $(p=0.011)$.

Conclusions: The IMA in rectal cancer patients enlarges as the TNM stage gets higher. The IMA diameter can be accepted as a possibly important marker for the staging of rectal cancer.
\end{abstract}

Key words: rectal cancer, tumor staging, inferior mesenteric artery.

\section{Introduction}

Colorectal cancer is one of the most commonly diagnosed cancers in the world [1, 2]. Rectal cancer accounts for nearly one third of the total incidence of colorectal cancer $[3,4]$. The inferior mesenteric artery (IMA) is one of the three unpaired branches of the abdominal aorta, which supplies the left side of the colon and rectum. It arises from the anterior aspect of the aorta below the renal artery branch points accompanied by the inferior mesenteric vein (IMV) [5]. For rectal cancer, the root of the IMA should be ligated in a standard procedure of total mesorectal excision [6, 7]. Although

\author{
Corresponding author: \\ Xiang Wang \\ Department of Vascular \\ Surgery \\ Taizhou Hospital of Zhejiang \\ Province \\ Zhejiang University \\ 150 Ximen Street \\ Linhai 317000, China \\ E-mail: doctorwx@yeah.net
}


several previous studies have shown a significantly increased IMV diameter in patients with rectal cancer $[8,9]$, no study has yet reported the IMA enlargement in rectal cancer. Thus, this study aimed to assess the association between the IMA diameter and rectal cancer.

\section{Material and methods}

\section{Patients}

The current study was approved by the ethics committee of Taizhou Hospital of Zhejiang Province, and all study participants provided informed consent. This study included a consecutive series of patients with primary rectal cancer in our center from July 2017 to June 2019. All patients underwent contrast enhanced computed tomography (CT) of the abdomen and pelvis after the presence of rectal cancer was confirmed by colonoscopic biopsy. Baseline patient characteristics, such as age, sex, weight, body mass index (BMI), and TNM stage ( $7^{\text {th }}$ AJCC classification), were retrospectively retrieved [10]. Patients were excluded if they had neoadjuvant treatment, synchronous colon cancer, history of abdominal surgery, and inflammatory bowel disease or severe atherosclerosis of the $a b$ dominal aorta branches, as these conditions might alter the IMA diameter. A control group of 42 patients with abdominal pain without rectal cancer or pathology in the IMA territory who underwent contrast enhanced $\mathrm{CT}$ of the abdomen and pelvis in the same time period was also selected.

\section{Imaging protocol and measurement of the inferior mesenteric artery diameter}

All patients underwent contrast enhanced $C T$ of the abdomen and pelvis on a 64-slice Toshiba Aquilion CT scanner using a standardized protocol. They were injected intravenously with $90 \mathrm{ml}$ of iopromide $(370$ mg/ml, Iopamiron 370; Bayer, Osaka,

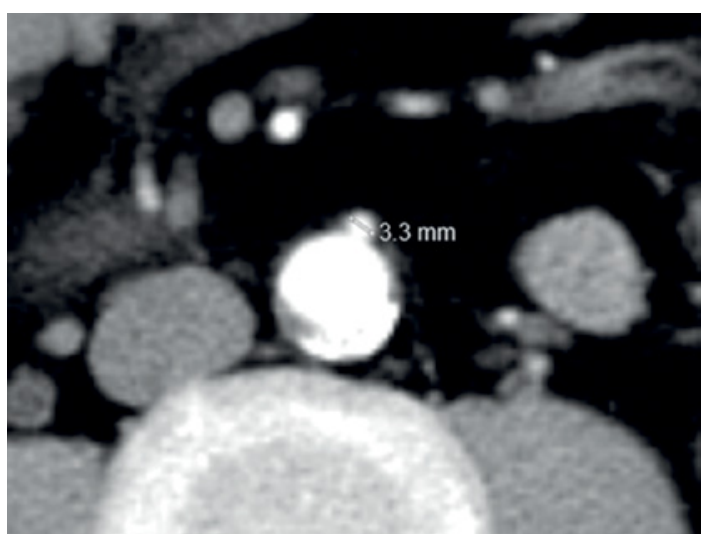

Figure 1. Measurement of the inferior mesenteric artery (IMA) on arterial phase axial computed tomography
Japan) at a speed of $3.0 \mathrm{ml} / \mathrm{s}$. Abdominal and pelvic scans were obtained using the following parameters: $120 \mathrm{kVp}, 220 \mathrm{mAs}$, and $0.5 \mathrm{~mm}$ slice thickness. The multiphase CT scanning protocol included unenhanced, arterial (30 s delay), portal venous (60 s delay), and equilibrium phases (120 s delay).

The arterial phase axial CT images were used to assess the IMA diameter. A single observer performed the measurements at the IMA origin using an electronic caliper on an imaging workstation where the images were magnified to $400 \%$ to increase accuracy (Figure 1). A second observer who was blinded to the former observer's results performed the measurements in the same way to confirm intra-observer agreement and reproducibility.

\section{Statistical analysis}

Data are presented as mean \pm standard deviation for continuous variables and as a number and percentage of the total for categorical variables. Student's t-test and the Mann-Whitney $U$ test were applied to evaluate continuous variables, and the $\chi^{2}$ test was performed to analyze categorical variables. The one-way analysis of variance and the least significant difference test were used for comparing continuous variables in multiple comparisons. The relationship among variables was assessed using the Pearson correlation coefficient. A $p$-value of less than 0.05 was used as the level of significance. All statistical analyses were performed using IBM SPSS Statistics version 22.0 (IBM Co., Armonk, NY, USA).

\section{Results}

In the period from July 2017 to June 2019, 150 patients were newly diagnosed with rectal cancer. Eleven patients receiving neoadjuvant treatment, 2 patients with a synchronous colon cancer and 2 patients with a history of abdominal surgery were excluded. Finally, 135 patients were enrolled in the patient group, 89 of whom were men and 46 women. Of the 135 patients, 21 patients who had presence of metastasis (M1) were classified as having stage IV cancers, while 114 patients who had absence of metastasis (MO) were adopted for surgical resection. Among the 114 surgical patients, according to the postoperative pathology, there were 28 patients with stage I cancers, 40 patients with stage II cancers, and 46 patients with stage III cancers. There were 4 patients with T1, 31 with T2, 51 with T3, and 28 with T4 tumors. Seventy-eight patients had NO tumors, and 46 patients had lymph node involvement (N1 = 34, $\mathrm{N} 2=12$ ). Forty-two patients were in the control group, with 18 women and 24 men.

There was no significant difference in sex, age, weight, or BMI between the patient group and 
Table I. Baseline patient characteristics

\begin{tabular}{|c|c|c|c|}
\hline Parameter & $\begin{array}{l}\text { Control } \\
\text { group } \\
(n=42)\end{array}$ & $\begin{array}{l}\text { Patient } \\
\text { group } \\
(n=135)\end{array}$ & $P$-value \\
\hline Age [years] & $61.6 \pm 9.9$ & $63.8 \pm 9.2$ & 0.211 \\
\hline \multicolumn{4}{|l|}{ Sex } \\
\hline Male & $24(57.1)$ & $89(65.9)$ & \multirow[t]{2}{*}{0.301} \\
\hline Female & $18(42.9)$ & $46(34.1)$ & \\
\hline Weight [kg] & $60.9 \pm 8.8$ & $61.1 \pm 9.6$ & 0.811 \\
\hline BMI $\left[\mathrm{kg} / \mathrm{m}^{2}\right]$ & $22.9 \pm 2.8$ & $23.7 \pm 2.9$ & 0.207 \\
\hline IMA diameter [mm] & $2.20 \pm 0.47$ & $2.49 \pm 0.53$ & $<0.001$ \\
\hline
\end{tabular}

$B M I$ - body mass index, IMA - inferior mesenteric artery

control group. However, we found that the mean IMA diameter was wider in the rectal cancer patients $(2.49 \pm 0.53 \mathrm{~mm})$ than in the control group $(2.20 \pm 0.47 \mathrm{~mm})$, which was statistically significant $(p<0.001)$ (Table I). There was an excellent correlation between the two observers in measuring the IMA diameter $(r=0.998, p<0.001)$ (Figure 2).

The IMA diameter of patients with stage I, stage II, stage III, and stage IV cancers was 2.24 $\pm 0.36 \mathrm{~mm}, 2.45 \pm 0.39 \mathrm{~mm}, 2.80 \pm 0.55 \mathrm{~mm}$, and $2.85 \pm 0.51 \mathrm{~mm}$, respectively $(p<0.001)$. Pearson analysis revealed a moderate, statistically significant linear relationship between IMA diameter and TNM stage $(r=0.519, p<0.001)$ (Figure 3$)$.

The IMA diameter of patients with T1, T2, T3, and T4 tumors was $2.18 \pm 0.31 \mathrm{~mm}, 2.39 \pm 0.50 \mathrm{~mm}$, $2.55 \pm 0.48 \mathrm{~mm}$, and $2.73 \pm 0.51 \mathrm{~mm}$, respectively $(p<0.001)$. A moderate, statistically significant linear relationship was also found between IMA diameter and T stage $(r=0.457, p<0.001)$ (Figure 4).

The IMA diameter of patients with NO, N1, and $\mathrm{N} 2$ tumors was $2.37 \pm 0.39 \mathrm{~mm}, 2.83 \pm 0.60 \mathrm{~mm}$, and $2.71 \pm 0.40 \mathrm{~mm}$, respectively $(p<0.001)$. However, we did not find a significant correlation between IMA diameter and $\mathrm{N}$ stage $(r=0.166$, $p=0.077$ ). Although patients with N1 and N2 tumors have a wider IMA diameter than patients with N0 tumors (N0 vs. N1, $p<0.001$; NO vs. N2, $p=0.020)$, there was no significant difference between patients with $\mathrm{N} 1$ and $\mathrm{N} 2$ tumors $(p=0.448)$. The IMA diameter of patients with M1 tumors was significantly wider than that in patients with MO tumors $(M O=2.54 \pm 0.51 \mathrm{~mm}$ vs. $\mathrm{M} 1=2.85$ $\pm 0.51 \mathrm{~mm}, p=0.011)$.

\section{Discussion}

To our knowledge, this is the first study to investigate the relationship between the IMA diameter and rectal cancer. Our findings reveal that the IMA in rectal cancer patients enlarges as the

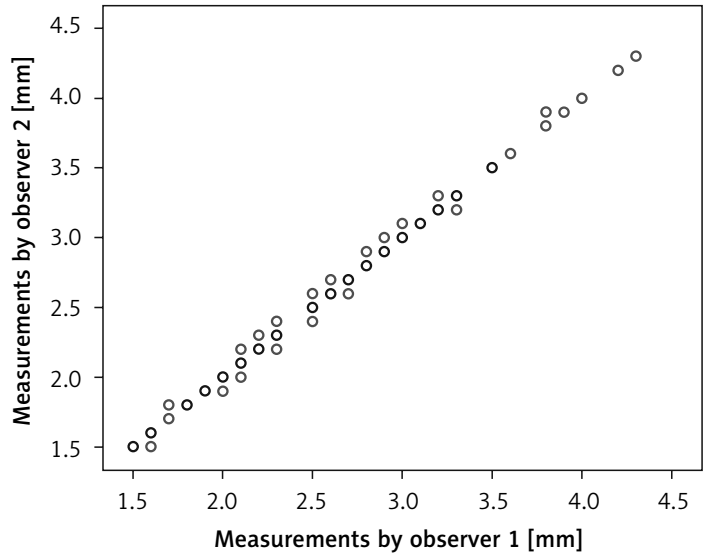

Figure 2. Intra-observer correlation plot for measurement of the inferior mesenteric artery (IMA) diameter. The correspondence between two observers was nearly perfect $(r=0.998, p<0.001)$

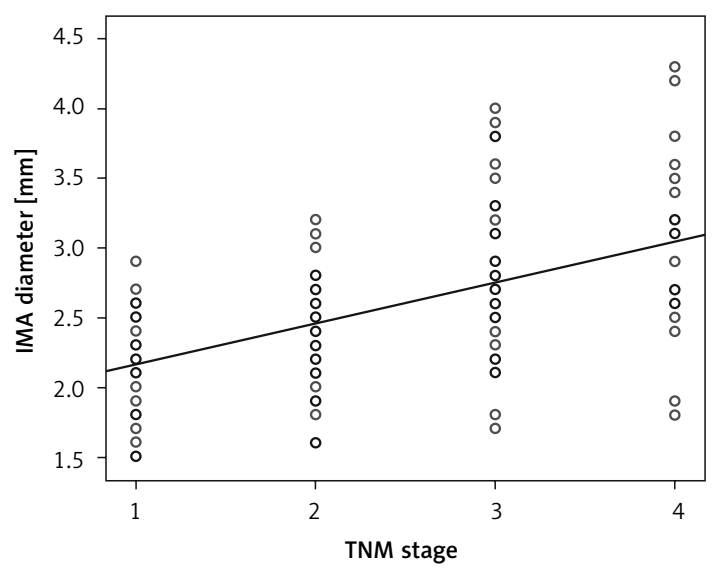

Figure 3. Correlation between TNM stage and inferior mesenteric artery (IMA) diameter. Pearson analysis showed a positive and moderate correlation between TNM stage and IMA diameter $(r=0.519$, $p<0.001$ ). TNM stages $1,2,3$, and 4 (X-axis) represent TNM stages I, II, III, and IV, respectively

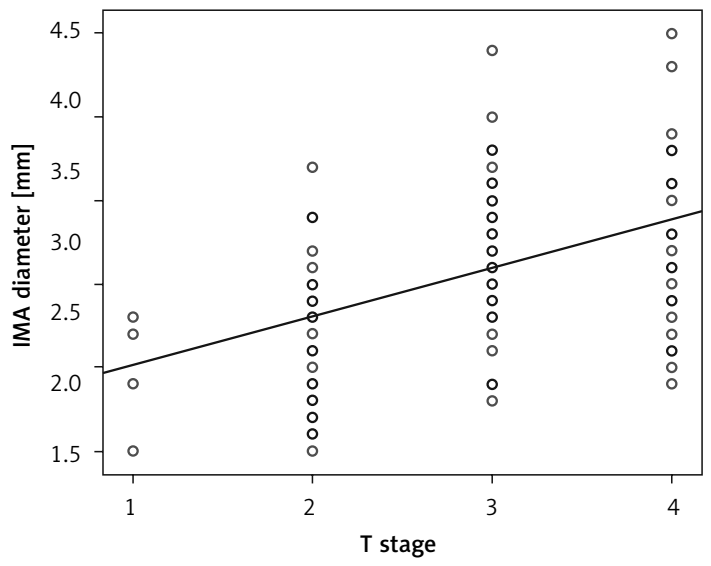

Figure 4. Correlation between $T$ stage and inferior mesenteric artery (IMA) diameter. Pearson analysis showed a positive and moderate correlation between T stage and IMA diameter $(r=0.457$, $p<0.001)$ 
TNM stage gets higher. The IMA diameter could be a potentially important marker for the staging of rectal cancer.

For rectal cancer, the optimal treatment strategies should be developed according to the stage of disease at patient presentation [11]. Patients with favorable prognostic features can proceed to total mesorectal excision directly. However, patients with poor prognosis tumors or metastasis usually require neoadjuvant chemoradiation therapy or palliative chemotherapy [12]. Magnetic resonance imaging (MRI) is the most accurate method to evaluate the local tumor extent of rectal cancer because of its high soft tissue contrast resolution [13]. MRI allows the precise assessment of the tumor relationship to the mesorectal fascia, which also defines the circumferential resection margin involvement in total mesorectal excision surgery [14]. Computed tomography is useful for systemic staging of rectal cancer, because it can examine the thorax, abdomen, and pelvis in a single examination. For evaluating the primary tumor, noninferiority of $\mathrm{CT}$, as compared with MRI, has been reported in several studies $[15,16]$. Additionally, CT is cheaper and more time-saving than MRI. For these reasons, CT has become a good alternative method for the staging of rectal cancer and influences the therapeutic approach to patients [17] In our hospital, CT of the thorax, abdomen, and pelvis is routinely performed in rectal cancer patients. In the present study, 15 patients were identified as requiring tumor downstaging on CT images and received neoadjuvant therapy. Distant metastases were identified in 21 patients. The remaining 114 patients underwent total mesorectal excision successfully.

Improvement in CT technologies has not only enabled accurate and non-invasive diagnoses of rectal cancer but also helped evaluate correlations between rectal cancer and vascular abnormity (increased blood flow and dilated blood vessels) [18]. Recently, changes in venous circulation have been observed in patients with colorectal cancer. Wu et al. reported that the IMV is significantly dilated in rectal cancer [19]. Khan et al. found a link between the diameter of the superior mesenteric vein and right colonic cancer [20]. Conventional angiography was commonly regarded as the gold standard for evaluating the IMA. However, with development in CT technologies, three-dimensional CT angiography has been suggested as an alternative modality for evaluating the IMA [5]. In fact, the IMA can be excellently visualized even on routine CT examinations. In our study, using a standard process, we managed to measure the IMA diameter precisely on the arterial phase axial CT images. As a result, the two observers showed perfect correlation in measurement.
As expected, our results showed that the IMA diameter of patients with rectal cancer was significantly wider than in those without rectal cancer. We further analyzed the IMA diameter of patients with different TNM stages and found that IMA diameter positively and moderately correlated with TNM stage $(r=0.519)$. Additionally, we found a positive and moderate relationship between IMA diameter and T stage $(r=0.457)$. Although we did not observe a correlation between IMA diameter and $\mathrm{N}$ stage, we found that the IMA diameter of the patients with $\mathrm{N} 1$ and N2 tumors was significantly larger than that of patients with NO tumors, and the IMA diameter of the patients with M1 tumors was significantly larger than that of patients with MO tumors. The main finding of these results is that the IMA diameter increases as the staging of rectal cancer gets higher. This finding suggests that the IMA diameter is a potential auxiliary marker for the staging of rectal cancer, which is important, because additional information for the staging of rectal cancer can benefit patients in better identifying whether they need neoadjuvant treatment [21].

The possible mechanism for our favorable results is believed to be angiogenesis [22]. It is well known that angiogenic chemical substances secreted by tumors lead to the formation of new vessels to supply tumors with oxygen and nutrients for their relentless growth [23-25]. The IMA dilatation may also be triggered by these substances directly. In the process of angiogenesis development, the IMA could enlarge to accommodate the increased blood flow caused by the increased number of tumor vessels. It is important to evaluate tumor angiogenesis because it is known to play an important role in tumor invasion and metastasis [26]. Currently, intratumoral microvascular density assessed by immunohistochemistry in archival tissue is a commonly used method to qualify the intensity of tumor angiogenesis [27]. An association between high microvascular density and poor prognosis in colorectal cancer has been reported by several studies using this method $[28,29]$. However, this method is limited to surgical patients whose resection specimens are available and cannot be used to predict or evaluate the response to neoadjuvant therapy or antiangiogenic therapy. Taking the above considerations and our findings together, we speculate that the IMA diameter has potential as a marker for prediction and evaluation of the effect of neoadjuvant treatment and antiangiogenic treatment.

The present study also has several limitations. First of all, this is a retrospective study, so there might have been selection bias. Furthermore, the samples are taken from a single center and thus are not large enough. The low number of pa- 
tients with stage T1 is another limitation. Additionally, the measurement error is also an important limitation. However, in our study, we used a standard process of measurement to minimize measurement error. We have demonstrated that the measurement results were reproducible.

In conclusion, our study provides an important finding that the IMA in rectal cancer patients enlarges as the TNM stage gets higher. The finding suggests that the IMA diameter can be considered as a potentially important marker for the staging of rectal cancer. However, future well-designed prospective studies to aid in evaluating this relationship are recommended.

\section{Conflict of interest}

The authors declare no conflict of interest.

\section{References}

1. Klimczak A, Miśkiewicz M, Mik M, Dziki A, Utada M, Kempińska-Mirosławska B. Colorectal cancer morbidity in Poland and Japan in the years 1999-2006 - a comparison. Arch Med Sci 2014; 10: 490-7.

2. Siegel RL, Miller KD, Fedewa SA, et al. Colorectal cancerstatistics 2017. CA Cancer J Clin 2017; 67: 177-93.

3. Klimczak A, Kempińska-Mirosławska B, Mik M, Dziki L, Dziki A. Incidence of colorectal cancer in Poland in 19992008. Arch Med Sci 2011; 7: 673-8.

4. Chen W, Zheng R, Baade PD, et al. Cancer statistics in China, 2015. CA Cancer J Clin 2016; 66: 115-32.

5. Murono K, Kawai K, Kazama S, et al. Anatomy of the inferior mesenteric artery evaluated using 3-dimensional CT angiography. Dis Colon Rectum 2015; 58: 214-9.

6. Fujii S, Ishibe A, Ota M, et al. Short-term and long-term results of a randomized study comparing high tie and low tie inferior mesenteric artery ligation in laparoscopic rectal anterior resection: subanalysis of the HTLT (high tie vs. low tie) study. Surg Endosc 2019; 33: 1100-10.

7. Janež J, Korać T, Kodre AR, Jelenc F, Ihan A. Laparoscopically assisted colorectal surgery provides better shortterm clinical and inflammatory outcomes compared to open colorectal surgery. Arch Med Sci 2015; 11: 1217-26.

8. Wu CC, Lee RC, Chang CY. Prediction of lymphovascular invasion in rectal cancer by preoperative CT. AJR Am J Roentgenol 2013; 201: 985-92.

9. Ivan CV, Mullineux JH, Verma R, et al. Assessment of the inferior mesenteric vein diameter as a surrogate marker to evaluate response to neoadjuvant chemoradiotherapy for locally advanced rectal adenocarcinoma. Colorectal Dis 2017; 19: 1076-80.

10. Edge SB, Compton CC. The American Joint Committee on Cancer: the 7th edition of the AJCC cancer staging manual and the future of TNM. Ann Surg Oncol 2010; 17: 1471-4.

11. Smith JJ, Garcia-Aguilar J. Advances and challenges in treatment of locally advanced rectal cancer. J Clin Oncol 2015; 33: 1797-808.

12. Glynne-Jones R, Wyrwicz L, Tiret E, et al.; ESMO Guidelines Committee. Rectal cancer: ESMO clinical practice guidelines for diagnosis, treatment and follow-up. Ann Oncol 2018; 29 (Suppl 4): iv263.

13. Brown G, Radcliffe AG, Newcombe RG, Dallimore NS, Bourne MW, Williams GT. Preoperative assessment of prognostic factors in rectal cancer using high-resolution magnetic resonance imaging. Br J Surg 2003; 90: 355-64.

14. Akasu T, linuma G, Takawa M, Yamamoto S, Muramatsu Y, Moriyama N. Accuracy of high-resolution magnetic resonance imaging in preoperative staging of rectal cancer. Ann Surg Oncol 2009; 16: 2787-94.

15. Ahmetoğlu A, Cansu A, Baki D, et al. MDCT with multiplanar reconstruction in the preoperative local staging of rectal tumor. Abdom Imaging 2011; 36: 31-7.

16. Sinha R, Verma R, Rajesh A, Richards CJ. Diagnostic value of multidetector row CT in rectal cancer staging: comparison of multiplanar and axial images with histopathology. Clin Radiol 2006; 61: 924-31.

17. Ahmetoğlu A, Cansu A, Baki D, et al. MDCT with multiplanar reconstruction in the preoperative local staging of rectal tumor. Abdom Imaging 2011; 36: 31-7.

18. Sun H, Xu Y, Yang Q, Wang W. Assessment of tumor grade and angiogenesis in colorectal cancer: wholevolume perfusion CT. Acad Radiol 2014; 21: 750-7.

19. Wu CC, Lee RC, Chang CY. Prediction of lymphovascular invasion in rectal cancer by preoperative CT. AJR Am J Roentgenol 2013; 201: 985-92.

20. Khan AN, Botchu R, Patel R, Elabassy M. Dilated SMV in colon cancer - is there any significance. J Gastrointest Cancer 2012; 43: 288-95.

21. Loos M, Quentmeier P, Schuster T, et al. Effect of preoperative radio(chemo)therapy on long-term functional outcome in rectal cancer patients: a systematic review and meta-analysis. Ann Surg Oncol 2013; 20: 1816-28.

22. Folkman J. Fundamental concepts of the angiogenic process. Curr Mol Med 2003; 3: 643-51.

23. McDougall SR, Anderson AR, Chaplain MA, Sherratt JA. Mathematical modelling of flow through vascular networks: implications for tumor-induced angiogenesis and chemotherapy strategies. Bull Math Biol 2002; 64: 673-702.

24. Sidiropoulou T, Karaferi A, Stachtos G, et al. Propofol but not sevoflurane decreases circulating levels of sEGFR and sE-selectin after colorectal cancer surgery. Arch Med Sci Civil Dis 2019; 4: e41-e50.

25. Lamami Y, Mesediyeva R, Arıkan S, et al. Preliminary report: one of the PD-1 gene variants may be a valuable marker for colorectal cancer. Arch Med Sci Civil Dis 2018; 3: e34-e40.

26. Ebos JM, Kerbel RS. Antiangiogenic therapy: impact on invasion, disease progression, and metastasis. Nat Rev Clin Oncol 2011; 8: 210-21.

27. Vermeulen PB, Gasparini G, Fox SB, et al. Quantification of angiogenesis in solid human tumours: an international consensus on the methodology and criteria of evaluation. Eur J Cancer 1996; 32: 2474-84.

28. Sökmen S, Sarioglu S, Füzün M, Terzi C, Küpelioglu A, Aslan B. Prognostic significance of angiogenesis in rectal cancer: a morphometric investigation. Anticancer Res 2001; 21: 4341-8.

29. Takebayashi Y, Akiyama S, Yamada K, Akiba S, Aikou T. Angiogenesis as an unfavorable prognostic factor in human colorectal carcinoma. Cancer 1996; 78: 226-31. 